\title{
Range Detection of the Extremely Low-Frequency Magnetic Field Produced by Laptop's AC Adapter
}

\author{
Darko Brodić ${ }^{1}$, Alessia Amelio ${ }^{2}$ \\ ${ }^{1}$ University of Belgrade, Technical Faculty in Bor, Vojske Jugoslavije 12, 19210 Bor, Serbia, dbrodic@tfbor.bg.ac.rs \\ ${ }^{2}$ DIMES University of Calabria, Via Pietro Bucci Cube 44, 87036 Rende (CS), Italy, aamelio@dimes.unical.it
}

\begin{abstract}
Human exposure to extremely low frequency magnetic field represents a risk to their health. This paper takes into consideration the level of an extremely low-frequency magnetic field between 30 and $300 \mathrm{~Hz}$ emitted by an AC laptop adapter. The experiment consists of testing 17 different AC adapters for laptops. During the testing, laptops are operated in a normal operating conditions as well as under heavy load. The magnetic field measurement is conducted in the area around the AC adapter. Obtained data is evaluated according to the critical level of the magnetic field proposed by safety standards. Furthermore, data is classified by a K-medians method in order to determine the critical levels of the magnetic field exposure in the nearby area of the AC adapter. Obtained classifications are evaluated according to safety standards, giving a critical analysis of magnetic field areas at risk. Due to emission of a very strong magnetic field in certain areas, a recommendation for safety use of the AC adapter is proposed.
\end{abstract}

Keywords: AC adapter, classification, dangerous levels, magnetic field, measurement.

\section{INTRODUCTION}

Magnetic fields can be generated by natural or man-made sources, which are typically a product of civilization [1]. These magnetic fields can be divided according to their characteristics. The naturally produced magnetic field, which is generated by the Earth, is characterized as a static one. On the contrary, the man-made sources determine a magnetic field with high peaks at different frequencies. It is especially noticeable for extremely low frequency (ELF) range, which is frequently encountered from electrical appliances. This frequency range is between 30 and $300 \mathrm{~Hz}$ [2]. Research has proven that an ELF magnetic field has certain negative impact on animals [3]. As a consequence, it can represent a potential hazard to human health.

Human exposure to the ELF magnetic field might cause some negative effects to their health [4]. Researchers have come to the conclusion that ELF magnetic fields can increase the risks of such illnesses as leukemia [5], brain cancer [6], amyotrophic lateral sclerosis [7] and Alzheimer's disease [8]. Furthermore, exposure to ELF magnetic field can have a negative influence on pregnant women, human fetus and small children [9], [10].

The reference level of the magnetic field exposure represents the critical level of the magnetic field emission above which the neighborhood of the emitter is unsafe for humans. The TCO (Tjänstemännens Centralorganisation) standard has proposed a reference level of $0.2 \mu \mathrm{T}$ in the frequency range up to $2 \mathrm{kHz}$ [11]. In contrast, ICNIRP
(International Commission on Non-Ionizing Radiation Protection) has suggested a more relaxed level of $0.2 \mathrm{mT}$ between 50 and $400 \mathrm{~Hz}$ [12]. In many other studies the reference level has been selected between $0.2 \mu \mathrm{T}$ and $0.4 \mu \mathrm{T}$ [9], [10], [13]. However, a problem still exists for a tested object that emits an ELF magnetic field close to the points where the magnetic field needs to be measured.

Today, laptops are the most widely used types of computers. This is so because of their portability. They represent not only a portable version of the desktop computer, but also a computer-based office on the road. New technologies used for their manufacturing contribute to making them more and more powerful and lighter as well. Furthermore, they are manufactured according to proposed standards like TCO for safe use and with materials that are almost $100 \%$ recyclable [11]. Although they are ecologically friendly, there still exists the problem of their safe use.

Essentially, the problem is linked to the use of electric power which generates an electric and magnetic field. The electric field can be more easily contained by an appropriate shielding than the magnetic field [14]. The magnetic field can be differentiated by its frequency. Different parts of laptops can generate electric and magnetic fields of different frequency. In this way, Wi-Fi, 3G/4G elements produce a very high frequency electromagnetic field, in the range between $2.4 \mathrm{GHz}$ and $6 \mathrm{GHz}$ for $\mathrm{Wi}-\mathrm{Fi}$, between $450 \mathrm{MHz}$ and $2 \mathrm{GHz}$ for $3 \mathrm{G}$, and between $2.3 \mathrm{GHz}$ and $5.8 \mathrm{GHz}$ for 
4G [15]. In contrast, other parts of laptops like the Central Processing Unit (CPU), Hard Disk (HD) or Solid State Disk (SSD) and memory produce an extremely low frequency magnetic field, below the range of $\mathrm{kHz}$. This part of the magnetic field is extremely dangerous due to its high level emission. Still, there is an additional element which is prerequisite for any laptop. It is an Alternating to Direct Current (AC/DC) adapter commonly called AC adapter.

The AC adapter represents an electronic device that supplies the laptop with electrical current and charges its battery. Hence, it should be technologically different from the typical computer supply because of its additional role of charging the laptop battery. Also, it should be compact, light and small in order to be the right companion for the laptop in its portability. It consists of a central unit which draws power from an outlet. Furthermore, the unit supplies the current to the laptop through the cord plugged into it.

An AC adapter is classified according to its power rating. It is measured in amperes, volts and/or watts. Typically, it is supplied with AC current at a voltage of 110 or $220 \mathrm{~V}$. Furthermore, an AC adapter creates an output with designated level of current, voltage and power specifically accustomed to each laptop. Today laptops typically use AC adapters that can supply from 30 to $125 \mathrm{~W}$.

In this paper, our attention is directed at the level of the magnetic field emission produced by the AC adapter in an extremely low frequency range. It is a very important topic to investigate because the AC adapter is an unavoidable addition to any laptop. The correct use of the AC adapter is of great importance to any laptop user. In this sense, we have tried to measure the magnetic field produced by AC adapters and further classify it into ranges of different dangerousness to laptop users. It has been established by Kmedians clustering according to the safe level limits proposed by different standards and in literature. In the end, we propose some precautions to safely use the AC adapter in the correct way. To the best of our knowledge, we are the first to investigate on this topic and to propose an automatic tool for measuring and classifying the magnetic field emissions of an AC adapter.

This paper is organized as follows. Section 2 gives a brief description of measuring devices. Section 3 describes the elements of the experiment. Section 4 presents the measurement results. Section 5 classifies the obtained results by K-medians clustering method and discusses the results. Section 6 draws a conclusion.

\section{MATERIALS AND METHODS}

\section{A. Magnetic field}

Around the emitter, like a laptop or an AC adapter, users are exposed to the emitted magnetic field. The measurement of the magnetic flux density $\mathbf{B}$ representing a vector can be split into 3 independent scalar fragments orthogonal to each other, i.e. $B_{x}, B_{y}$ and $B_{z}$ in the direction of the axes $x, y$ and $z$.

$$
\mathbf{B}(\mathrm{r}, \mathrm{t})=\mathrm{B}_{\mathrm{x}}(\mathrm{t}) \cdot \mathbf{x}+\mathrm{B}_{\mathrm{y}}(\mathrm{t}) \cdot \mathrm{y}+\mathrm{B}_{\mathrm{z}}(\mathrm{t}) \cdot \mathbf{z},
$$

where $t$ is the time, and $\mathbf{r}$ is the position vector. This vector can be split into 3 independent vectors $\mathbf{x}, \mathbf{y}$ and $\mathbf{z}$. At a given time point, eq. (1) becomes:

$$
\mathbf{B}(\mathbf{r})=B_{x} \cdot \mathbf{x}+B_{y} \cdot \mathbf{y}+B_{z} \cdot \mathbf{x} .
$$

Measuring devices measure the scalar components of the magnetic flux density $\mathbf{B}$, i.e. $B_{x}, B_{y}$ and $B_{z}$, and its root mean square (RMS) B equal to:

$$
B=\sqrt{B_{x}^{2}+B_{y}^{2}+B_{z}^{2}} .
$$

In the case of magnetic field at multiple frequencies, the total magnetic flux density $B_{\text {tot }}$ is defined as:

$$
B_{t o t}=\sqrt{\sum_{i=1}^{n} B_{i}},
$$

where $B_{i}$ is the RMS of the magnetic flux density at frequency $i$, and $n$ is the total number of frequencies.

\section{B. Measuring devices}

We performed the ELF magnetic field measurement by measuring device Lutron EMF-828 with separate probe [16]. The device measures the magnetic flux density from $0.01 \mu \mathrm{T}$ to $2 \mathrm{mT}$ in the frequency range from 30 to $300 \mathrm{~Hz}$. It can be used for three measurement extents: $20 \mu \mathrm{T}, 0.2 \mathrm{mT}$ and $2 \mathrm{mT}$. The precision of the measurement is in the order of $0.01 \mu \mathrm{T}$.

The ELF magnetic field is also tested with the measuring device Aaronia Spectran NF-5035 [17], which has a frequency range from $1 \mathrm{~Hz}$ to $1 \mathrm{MHz}$. This measurement confirms the results obtained from Lutron EMF-828. Fig.1. shows the measuring devices Aaronia Spectran NF-5035 and Lutron EMF-828.

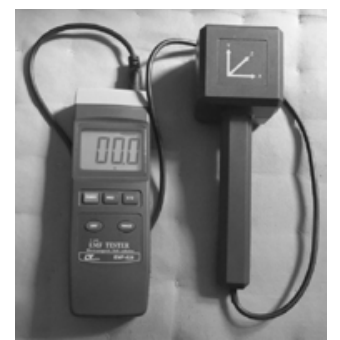

a)

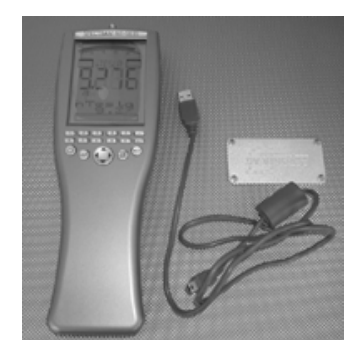

b)
Fig.1. The ELF measuring devices: a) Lutron EMF-828, and b) Aaronia Spectran NF-5035.

\section{EXPERIMENT}

The experiment was conducted on 17 different laptop AC adapters. We proposed 6 measurement positions at which an ELF magnetic field was measured. They were at the top and at the bottom of the AC adapter and $30 \mathrm{~cm}$ away from these 
positions in four directions which were under $90^{\circ}$ to each other. Fig.2. shows the measurement positions.

Furthermore, we tested the AC adapter work in two different operating conditions:

1. Under normal operating conditions, which includes working with office applications, web browsing, etc. All 17 laptops can work in normal operating conditions. Hence, the results from all 17 laptop AC adapters were obtained.

2. Under stress operating condition, using Futuremark 3DMARK Vantage [18] testing program for graphics intensive applications in ref. [19]. Only 9 out of 17 laptops are able to run 3DMARK Vantage. Hence, the results have been obtained for the first 9 out of 17 AC adapters.

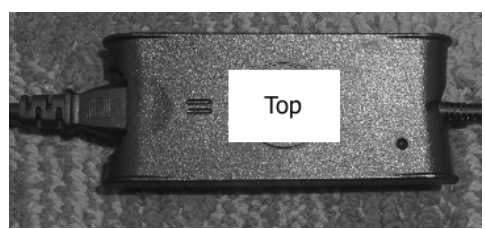

a)

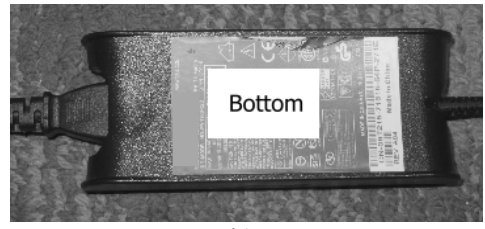

b)

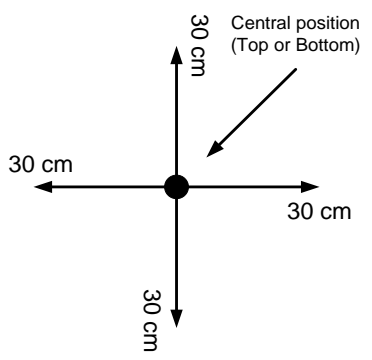

C)

Fig.2. Measurement positions of the AC adapter: a) Top position, b) Bottom position, c) Away positions.

\section{MEASURING RESULTS}

It can be noticed that the level of the measured magnetic field, which is $30 \mathrm{~cm}$ away from the top or bottom positions, is around $0.01 \mu \mathrm{T}$. This means that it represents a background magnetic field. Hence, it may be considered as negligible. The results of the measured ELF magnetic field at the top and bottom of the AC adapter in a normal operating condition for all 17 laptops are given in Table 1.

Table 1. includes the following measures: (i) Average, (ii) Difference and (iii) $\Delta$ defined as:

$$
\begin{gathered}
\text { Average }=\frac{E M F(\text { top })+E M F(\text { bottom })}{2}, \\
\text { Difference }=\mid E M F(\text { top })-E M F(\text { bottom }) \mid,
\end{gathered}
$$

$$
\Delta=\text { Difference / EMF(top) }
$$

The magnetic field measured at the top of AC adapters has a minimum value of $0.03 \mu \mathrm{T}$, while the maximum value reaches $11.17 \mu \mathrm{T}$. Similarly, at the bottom of an AC adapter the obtained minimum value of the measured magnetic field is $0.02 \mu \mathrm{T}$, while the maximum has a value of $19.75 \mu \mathrm{T}$. If we look carefully at the data, only one AC adapter emits a magnetic field value which is in the safe region.

Table 1. Measured ELF magnetic field at the top and bottom positions of the AC adapters for laptops running in normal conditions.

\begin{tabular}{|c|c|c|c|c|c|}
\hline $\begin{array}{c}\text { Laptop } \\
\text { No. }\end{array}$ & $\begin{array}{c}\text { Top } \\
{[\mu \mathrm{T}]}\end{array}$ & $\begin{array}{c}\text { Bottom } \\
{[\mu \mathrm{T}]}\end{array}$ & $\begin{array}{c}\text { Average } \\
{[\mu \mathrm{T}]}\end{array}$ & $\begin{array}{c}\text { Difference } \\
{[\mu \mathrm{T}]}\end{array}$ & $\begin{array}{c}\Delta \\
{[\%]}\end{array}$ \\
\hline L1 & 1.17 & 0.82 & 0.99 & 0.35 & 30.03 \\
\hline L2 & 1.76 & 19.75 & 10.76 & 17.99 & 1019.85 \\
\hline L3 & 0.56 & 1.84 & 1.20 & 1.28 & 229.81 \\
\hline L4 & 10.10 & 11.77 & 10.93 & 1.66 & 16.48 \\
\hline L5 & 2.62 & 4.71 & 3.67 & 2.09 & 79.50 \\
\hline L6 & 2.93 & 4.94 & 3.94 & 2.01 & 68.39 \\
\hline L7 & 4.39 & 5.40 & 4.90 & 1.01 & 22.90 \\
\hline L8 & 1.11 & 3.88 & 2.50 & 2.77 & 249.67 \\
\hline L9 & 6.29 & 1.17 & 3.73 & 5.12 & 81.42 \\
\hline L10 & 2.26 & 0.65 & 1.45 & 1.60 & 71.05 \\
\hline L11 & 0.57 & 2.03 & 1.30 & 1.46 & 257.30 \\
\hline L12 & 0.51 & 5.72 & 3.12 & 5.21 & 1020.46 \\
\hline L13 & 0.49 & 6.45 & 3.47 & 5.96 & 1210.39 \\
\hline L14 & 11.17 & 7.20 & 9.19 & 3.97 & 35.54 \\
\hline L15 & 2.26 & 0.65 & 1.45 & 1.60 & 71.05 \\
\hline L16 & 1.79 & 3.71 & 2.75 & 1.92 & 106.95 \\
\hline L17 & 0.03 & 0.02 & 0.03 & 0.01 & 18.33 \\
\hline
\end{tabular}

Furthermore, if we average the top and bottom magnetic field values, we can get a more uniform result: $2.94 \mu \mathrm{T}$ at the top and $4.75 \mu \mathrm{T}$ at the bottom of the AC adapter. Obviously, an AC adapter is designed mainly to emit a lower magnetic field at the top, which means that the position of the AC adapter matters. This is mainly because the circuitry where current flows and where inner components are located is positioned at the bottom of the device. Later, the inclusion of an absolute difference between the top and bottom magnetic field measured value brings new information to be discussed later. The absolute differences are in a range between $0.01 \mu \mathrm{T}$ and $17.99 \mu \mathrm{T}$. It can be noticed that AC adapters emitting higher peaks at any position (top or bottom), typically have a much lower magnetic field emission in the opposite position. This creates extremely high values of differences, which again confirms that the AC adapter is designed to have one "right" position. Obviously, the other position can be considered as a "wrong" position. For this reason, it is convenient to put the "wrong" position of the device on the table, and the "right" position in front of the user. Accordingly, when the device is hanging freely, it is suitable to position its "right" side in front of the user. It is a very important conclusion for the laptop user on how to properly position the AC adapter. 
As a confirmation of the previous discussion, we can compute the measure $\Delta$ given in $\%$ which defines the relative deviation of the measured magnetic field values at the top and at the bottom of the AC adapter. The obtained values are between $16.48 \%$ and $1210.39 \%$. As a final conclusion, which can be drawn from the results presented in Table 1., it is clear that in spite of differences between AC adapters the level of the measured magnetic field is lower at the top than at the bottom.

Table 2. shows the results of the measured ELF magnetic field at the top and bottom of the AC adapter for the first 9 laptops that operate under stress condition. In this case, only the first 9 out of 17 laptops have passed the test of Futuremark 3DMARK Vantage [18].

Table 2. Measured ELF magnetic field at the top and bottom positions of the AC adapters for laptops running under stress conditions.

\begin{tabular}{|c|c|c|c|c|c|}
\hline $\begin{array}{c}\text { Laptop } \\
\text { No. }\end{array}$ & $\begin{array}{c}\text { Top } \\
{[\mu \mathrm{T}]}\end{array}$ & $\begin{array}{c}\text { Bottom } \\
{[\mu \mathrm{T}]}\end{array}$ & $\begin{array}{c}\text { Average } \\
{[\mu \mathrm{T}]}\end{array}$ & $\begin{array}{c}\text { Difference } \\
{[\mu \mathrm{T}]}\end{array}$ & $\begin{array}{c}\Delta \\
{[\%]}\end{array}$ \\
\hline L1 & 5.57 & 3.77 & 4.67 & 1.80 & 32.33 \\
\hline L2 & 2.79 & 20.95 & 11.87 & 18.15 & 649.37 \\
\hline L3 & 1.28 & 5.09 & 3.18 & 3.81 & 298.60 \\
\hline L4 & 12.52 & 5.44 & 8.98 & 7.08 & 56.52 \\
\hline L5 & 1.33 & 1.71 & 1.52 & 0.38 & 28.21 \\
\hline L6 & 1.09 & 13.98 & 7.54 & 12.89 & 1178.94 \\
\hline L7 & 3.76 & 12.47 & 8.12 & 8.71 & 231.36 \\
\hline L8 & 2.15 & 5.32 & 3.73 & 3.17 & 147.49 \\
\hline L9 & 18.80 & 7.20 & 13.00 & 11.60 & 61.71 \\
\hline
\end{tabular}

Hence, valid results are obtained for AC adapters of the first 9 laptops. The obtained magnetic field values at the top of AC adapters are between $1.09 \mu \mathrm{T}$ and $18.80 \mu \mathrm{T}$. Also, the measured magnetic field values at the bottom of AC adapters are from 1.7108 to $20.9508 \mu \mathrm{T}$. These magnetic field values are higher than those previously obtained in normal operating conditions. Hence, stress condition by intensive graphic programs contributes to higher levels of magnetic field emission. Furthermore, if we average the results of the measured magnetic field obtained from the top and bottom of AC adapters, then their minimum is $1.52 \mu \mathrm{T}$, while their maximum is $13.00 \mu \mathrm{T}$. Again, the level of the emitted magnetic field is increased by a margin of at least $20 \%$. Furthermore, the absolute difference receives similar values, i.e. from $0.38 \mu \mathrm{T}$ to $18.15 \mu \mathrm{T}$. Hence, we can conclude that the modality of an operating AC adapter is similar to normal operating conditions leading to a similar efficiency. This point is confirmed by the value of the $\Delta$ measure which is between 28.21 and $1178.94 \%$. However, in spite of similar efficiency, the AC adapter is under higher power consumption when the laptop operates under stress conditions. Essentially, it dissipates power, achieving the highest level of magnetic field emission in its neighborhood. This can be seen in terms of higher power dissipation.

\section{ClassificAtion}

From the laptop user point of view, the most important questions are: (i) What is the level of emitted ELF magnetic field around an AC adapter? (ii) What is the correct way of using the AC adapter? (iii) What is a safe distance from the AC adapter? and (iv) What can be used to minimize safety risks?

To answer all these questions, we can compare the results obtained from measurements of the ELF magnetic field around an AC adapter and use a clustering tool to establish the safety and/or danger zones in order to correctly use the AC adapter.

Each measuring position (top or bottom) of the AC adapter is represented by a one-dimensional feature, which is the measured magnetic field at that position. Consequently, we realize 4 different datasets. The first one (named as top normal) and second one (named as bottom normal) contain the 17 features respectively from the top and bottom part of the AC adapters in normal operating conditions. The third one (named as top stress) and the fourth one (named as bottom stress) include 9 features respectively from the top and bottom part of the AC adapters under stress operating conditions. Each dataset is subjected to a K-medians clustering method to detect magnetic field ranges corresponding to different levels of magnetic fields emitted from AC adapters.

\section{A. The method}

K-medians is a center-based clustering method [20] producing compact clusters and quite robust to outliers. We adopted K-medians because it is particularly apt to deal with one-dimensional measured data [21], [22], [23]. Furthermore, a test has been conducted concerning the application of different clustering and discretization algorithms on magnetic field values emitted from an AC adapter. Tests revealed that K-medians produces the best results in terms of ranges below and above the safety limit of $0.2 \mu \mathrm{T}$. The number $\mathrm{K}$ of output clusters is an input parameter. K-medians considers each cluster represented by its centroid, which is the median value of data inside that cluster. The algorithm consists of a few easy steps: (i) centroids initialization, (ii) data assignment to centroids, and (iii) centroids re-computation. In the first step, $\mathrm{K}$ centroids are selected to create initial clusters. This is a critical step, because the selection of initial centroids can influence the final solution. In the second step, each data is assigned to that centroid having the smallest $\mathrm{L}_{1}$ norm from it. Finally, centroids are computed again for each cluster as the median value of data inside that cluster. Steps (ii) and (iii) are repeated multiple times and centroids change their position, until no movements are performed and centroids become stable. K-medians algorithm aims to minimize the following function:

$$
J=\sum_{j=1}^{k} \sum_{i=1}^{n}\left\|x_{i}^{(j)}-c_{j}\right\|,
$$

where $\left\|x_{i}^{(j)}-c_{j}\right\|$ is the $\mathrm{L}_{1}$ norm between the measured value $x_{i}{ }^{(j)}$ in cluster $j$ and the centroid $c_{j}$ in cluster $j$. 


\section{B. Results and discussion}

Looking at measured values at the top part in Table 1., we notice that only one value, i.e from Laptop 17 , is $0.03 \mu \mathrm{T}$, is below the reference limit of $0.2 \mu \mathrm{T}$. Similarly, at the bottom parts in Table 1., we have only one value below $0.2 \mu \mathrm{T}$, i.e. from Laptop 17, which is $0.02 \mu \mathrm{T}$. Furthermore, observing the general distribution of top and bottom measured values in normal operating conditions in Table 1., we notice that 4 magnetic field ranges are received. Then, we confirm the optimal $\mathrm{K}$ value equal to 4 by experimental evaluation, performed on top normal and bottom normal datasets. In particular, we run K-medians algorithm on the two datasets at different values of a $\mathrm{K}$ parameter, from 2 to 5 . In this way, we found that the $\mathrm{K}$ value determining the best result on both the datasets is 4 , because only in this case the low values of $0.03 \mu \mathrm{T}$ and $0.02 \mu \mathrm{T}$ are correctly isolated from the other values in both datasets, according to the TCO reference limit of $0.2 \mu \mathrm{T}$. Our aim is to correctly separate safe from unsafe measured values (lower and higher than $0.2 \mu \mathrm{T}$ ), we set the $\mathrm{K}$ value of $\mathrm{K}$-medians algorithm to 4 , in order to receive: (i) low, (ii) middle, (iii) high, and (iv) very high ranges. Association between classifications (low, middle, high and very high) and ranges is performed by considering the maximum and minimum measured values inside each range. Furthermore, the same $\mathrm{K}$ value is adopted on top and bottom stress datasets.

The clustering algorithm has been implemented in Matlab R2015a. Clustering experimentation was performed on a laptop computer with Quad-core CPU $2.2 \mathrm{GHz}, 16 \mathrm{~GB}$ RAM and UNIX operating system. Because K-medians initialization influences the final solution, for each dataset, the algorithm has been run 50 times, each time with a new set of initial centroids. A clustering solution determining the lowest value of $J$ function is given as output of the procedure. Application of K-medians algorithm on the top and bottom normal datasets generates the magnetic field ranges reported in Table 3 .

It is clearly visible that algorithm determines wellseparated ranges from both top and bottom parts of an AC adapter in normal operating conditions. It is also observable that middle and high ranges exhibit minimum and maximum values which are much higher than values of the same ranges obtained from laptop measurement [24], [25], [26]. This is a typical condition of AC adapters and depends on the different architecture of the electrical device. Top and bottom parts emit at a very similar low range, with maximum values of $0.03 \mu \mathrm{T}$ and $0.02 \mu \mathrm{T}$ respectively, perfectly discriminated from the algorithm, according to the safety reference limit. On the contrary, the bottom part exhibits different ranges of higher value than the top part. In fact, the top part reaches a middle range with the highest peak of $1.17 \mu \mathrm{T}$, while the bottom part emits at a middle range with the highest peak of $2.03 \mu \mathrm{T}$. Again, the top part emits at high range with a minimum of $1.79 \mu \mathrm{T}$, differently from the bottom part having a minimum of $3.71 \mu \mathrm{T}$. Also, the top part has the highest peak of $4.40 \mu \mathrm{T}$, while the bottom part reaches $11.77 \mu \mathrm{T}$. Finally, a strong difference appears in a very high range, in which the top part reaches the maximum value of $11.18 \mu \mathrm{T}$ while the bottom part reaches the minimum value of $19 \mu \mathrm{T}$.
Table 4. shows the magnetic field ranges obtained from a K-medians algorithm on top and bottom stress datasets. Under stress operating conditions, the bottom part of AC adapter emits a stronger magnetic field level than the top part. In fact, the top part reaches the highest peak in the middle range at $2.15 \mu \mathrm{T}$, while the bottom part reaches the highest peak at $7.20 \mu \mathrm{T}$. The difference is noticeable for high range, in which the top part exhibits a value between $2.79 \mu \mathrm{T}$ and $5.57 \mu \mathrm{T}$, while the bottom part between $12 \mu \mathrm{T}$ and $14 \mu \mathrm{T}$. Finally, the top part emits at a very high range with a maximum value of $18.80 \mu \mathrm{T}$, while the bottom part has a minimum value of $20 \mu \mathrm{T}$ in the very high range.

Table 3. Magnetic field ranges emitted at top and bottom positions of the AC adapters for laptops running in normal operating conditions.

\begin{tabular}{|c|c|c|c|}
\hline \multicolumn{2}{|c|}{ Top Normal } & \multicolumn{2}{c|}{ Bottom Normal } \\
\hline $\begin{array}{c}\text { Magnetic } \\
\text { field ranges }\end{array}$ & $\begin{array}{c}\text { min-max } \\
\text { values }(\mu \mathrm{T})\end{array}$ & $\begin{array}{c}\text { Magnetic } \\
\text { field ranges }\end{array}$ & $\begin{array}{c}\text { min-max } \\
\text { values }(\mu \mathrm{T})\end{array}$ \\
\hline low & $0.00-0.03$ & low & $0.00-0.02$ \\
\hline middle & $0.49-1.17$ & middle & $0.65-2.03$ \\
\hline high & $1.79-4.40$ & high & $3.71-11.77$ \\
\hline very high & $6.29-11.18$ & very high & $>19.00$ \\
\hline
\end{tabular}

Table 4. Magnetic field ranges emitted at top and bottom positions of the AC adapters for laptops running under stress operating conditions.

\begin{tabular}{|c|c|c|c|}
\hline \multicolumn{2}{|c|}{ Top Stress } & \multicolumn{2}{c|}{ Bottom Stress } \\
\hline $\begin{array}{c}\text { Magnetic } \\
\text { field ranges }\end{array}$ & $\begin{array}{c}\text { min-max } \\
\text { values }(\mu \mathrm{T})\end{array}$ & $\begin{array}{c}\text { Magnetic } \\
\text { field ranges }\end{array}$ & $\begin{array}{c}\text { min-max } \\
\text { values }(\mu \mathrm{T})\end{array}$ \\
\hline middle & $1.09-2.15$ & middle & $1.71-7.20$ \\
\hline high & $2.79-5.57$ & high & $12.00-14.00$ \\
\hline very high & $12.52-18.80$ & very high & $>20.00$ \\
\hline
\end{tabular}

Again, looking at Table 3. and Table 4., a comparison is performed between ranges in normal operating conditions and under stress. In particular, we can observe that the top part of an AC adapter emits at higher magnetic field levels when it operates under stress conditions rather than in normal conditions. It is confirmed from the highest peak of the middle range, which is $1.17 \mu \mathrm{T}$ in normal conditions and $2.15 \mu \mathrm{T}$ under stress conditions. Also, minimum and maximum of high range reach values of $2.79 \mu \mathrm{T}$ and $5.57 \mu \mathrm{T}$ respectively under stress conditions, while in normal conditions they are lower and respectively of $1.79 \mu \mathrm{T}$ and $4.40 \mu \mathrm{T}$. The difference is noticeable in the very high range, having a value of $6.29 \mu \mathrm{T}-11.18 \mu \mathrm{T}$ in normal conditions, and a much higher value of $12.52 \mu \mathrm{T}$ $18.80 \mu \mathrm{T}$ under stress conditions.

A similar consideration can be observed for the bottom part of the AC adapter. In fact, the highest peak of the middle range is $2.03 \mu \mathrm{T}$ in normal conditions, differently from the value of $7.20 \mu \mathrm{T}$ under stress conditions. It is also visible in high range, with a peak of $11.77 \mu \mathrm{T}$ in normal conditions, and of $14 \mu \mathrm{T}$ under stress conditions. Finally, very high range includes values higher than $19 \mu \mathrm{T}$ in normal conditions, and values higher than $20 \mu \mathrm{T}$ under stress conditions. 
An important conclusion of this analysis is that the bottom part of the AC adapter emits at stronger magnetic field levels than its top part. Again, the ranges of the magnetic field of an AC adapter are higher when the laptop is operating under stress conditions than when it runs in normal operating conditions. Finally, the most unsafe conditions for a laptop user are when the laptop is operating under stress conditions and the bottom part of the AC adapter is in direct or close contact with the user.

An analysis of the ranges is very important, but not sufficient to assess the emission degree of AC adapters. To clearly understand other critical aspects, it is essential to know the distribution of top/bottom parts of AC adapters emitting in each range.

Fig.3. shows the composition of the magnetic field ranges obtained on top normal dataset.

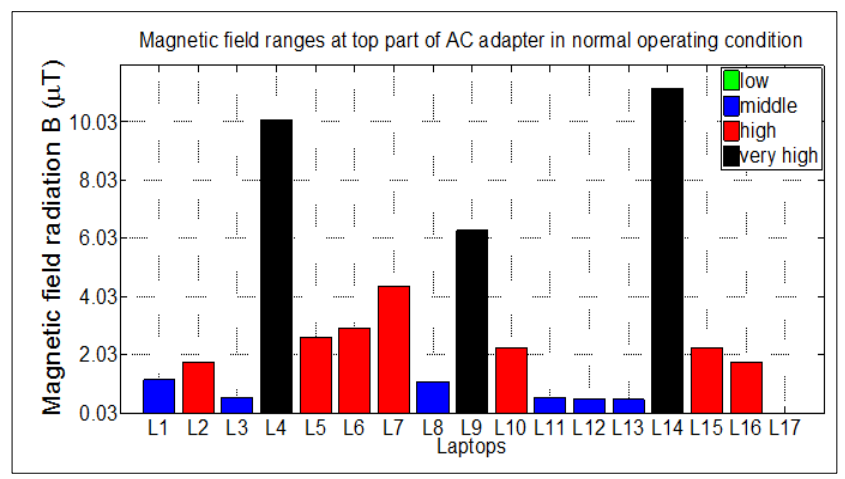

Fig.3. Clustering results corresponding to the magnetic field ranges emitted at the top part of AC adapters when laptops operate in normal conditions. Vertical bars represent the 17 laptops (L1,...,L17) with the top measured value.

We observe that only one AC adapter is included inside the low magnetic field range (Laptop 17), while all other AC adapters are included inside the middle, high and very high ranges. In particular, around $40 \%$ of the top parts of AC adapters emit at high range, while around $35 \%$ of them emit at middle range, which is above the reference limit of $0.2 \mu \mathrm{T}$, and around $18 \%$ of them emit at very high range.

Fig.4. illustrates the composition of magnetic field ranges obtained on the bottom normal dataset. Also in this case, only one AC adapter is included in low range (Laptop 17). However, we observe that around $53 \%$ of the bottom parts of AC adapters emit at high range, around $35 \%$ of them emit at middle range, and one bottom part of an AC adapter emits at very high range (Laptop 2). Consequently, also in this case, around $94 \%$ of the bottom parts of AC adapters emit at ranges above the safety reference limit of $0.2 \mu \mathrm{T}$.

Fig.5. depicts the composition of magnetic field ranges obtained on top stress dataset. In this case, low range is empty and consequently not reported. Also, around $55 \%$ of the top parts of AC adapters emit at high and very high ranges and around $45 \%$ of the top parts of AC adapters emit in the middle range. This indicates that $10 \%$ more of the top parts of AC adapters emit at a strong magnetic field range wrt the normal operating condition.

Finally, Fig.6. reports the composition of magnetic field ranges obtained on bottom stress dataset. Also in this case, low range is not reported because no AC adapters emit in low range. Although around $67 \%$ of laptop AC adapters are included inside middle range, the inner magnetic field values are very high and far above the limit of $0.2 \mu \mathrm{T}$. Again, around $30 \%$ of AC adapters emit in high and very high ranges.

This analysis reveals that a large percentage of laptop AC adapters measured on their top and bottom parts emit at middle, high and very high magnetic field levels, and that very few of them emit at low level. Furthermore, middle, high and very high ranges outweigh the reference safety limit of $0.2 \mu \mathrm{T}$. Hence, users should possibly be cautious in managing the AC adapter of laptops. In particular, a safety distance of $30 \mathrm{~cm}$ from the center point of an AC adapter should be observed when users operate with a laptop.

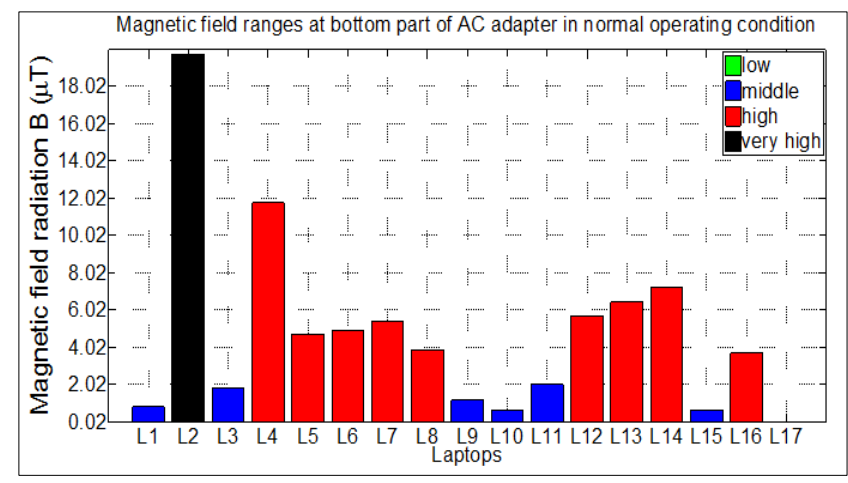

Fig.4. Clustering results corresponding to the magnetic field ranges emitted at the bottom part of AC adapters when laptops operate in normal conditions. Vertical bars represent the 17 laptops (L1,...,L17) with the bottom measured value.

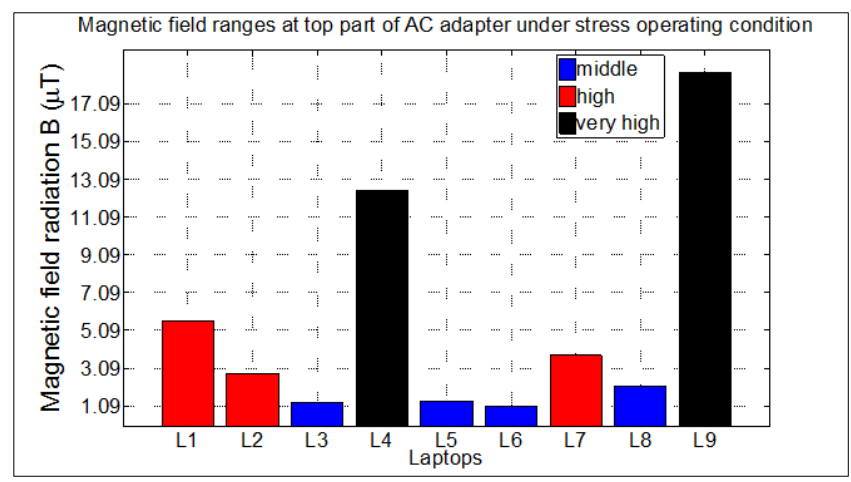

Fig.5. Clustering results corresponding to the magnetic field ranges emitted at the top part of AC adapters when laptops operate under stress conditions. Vertical bars represent the first 9 laptops (L1,...,L9) with the top measured value.

Also, because one of the two parts of AC adapter emits at a higher level of magnetic field (bottom part) than the other part (top part), it is recommended for the users to be in direct contact only with the top part of the AC adapter, when he/she touches it with feet, hands, fingers or other parts of the body. Again, when a laptop operates under stress conditions, that is when a laptop is overloaded with heavy programs, users should avoid contact with the AC adapter of 
the laptop. In fact, we have demonstrated that under stress conditions of the laptop, its AC adapter emits magnetic field levels whose prolonged exposition could put at risk human health. Therefore, it is recommended for laptop users to observe these easy safety rules for AC adapters. But, more importantly, it is a suggestion also for laptop manufacturers, who are invited to produce low emission AC adapters as well as checking that the top side of the AC adapter always emits a lower level of ELF magnetic field than bottom side. In this sense, manufacturers should create AC adapters with smaller ELF magnetic field emission at their top. Essentially, the solution (in some cases) can be very easy, i.e. changing only the position of the top and bottom plastic elements. In this way, the changing of the AC adapter sides (top to bottom) will enable lower emission of the ELF magnetic field produced by the AC adapter.

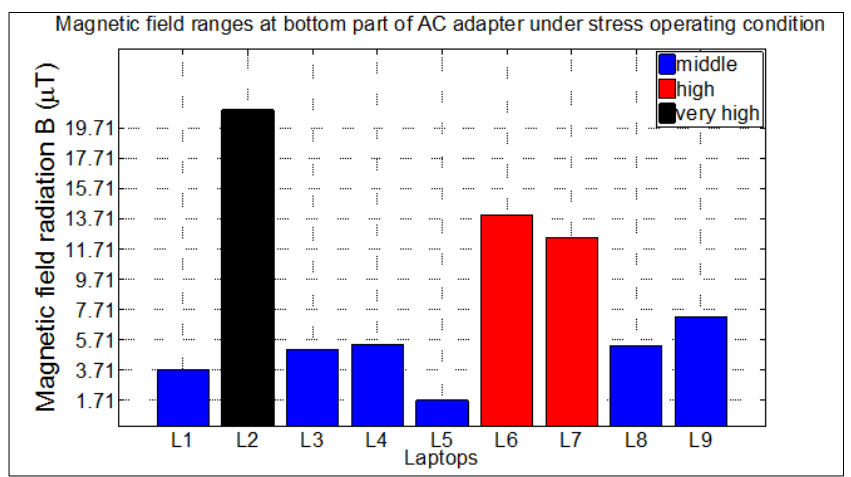

Fig.6. Clustering results corresponding to the magnetic field ranges emitted at the bottom part of AC adapters when laptops operate under stress conditions. Vertical bars represent the first 9 laptops (L1,...,L9) with the bottom measured value.

\section{CONCLUSION}

This paper has addressed the problems related to the ELF magnetic field emitted from the AC adapter, which is an infallible companion to the laptop. The measurement of the ELF magnetic field was performed by Lutron EMF-828 and AARONIA NF-5035 devices. The measured results showed a high emission rate of the magnetic field in the area around the AC adapter. To clearly evaluate the results, data has been clustered by a K-medians algorithm in order to differentiate magnetic field levels determining a risk to laptop users. Classification shows that almost all AC adapters emitted a very strong magnetic field. Hence, AC adapters should be used with caution. To employ them safely, we propose putting the AC adapters at least $30 \mathrm{~cm}$ away from any part of the human body.

\section{APPENDIX}

\section{A. TCO standard excerpt}

TCO'05 proposed a measurement methodology of magnetic field RMS value in two frequency ranges: (i) band 1 with a frequency range between $50 \mathrm{~Hz}$ and $2 \mathrm{kHz}$, and (ii) band II with a frequency range between $2 \mathrm{kHz}$ and $400 \mathrm{kHz}$ [11]. TCO measuring geometry includes measurement positions, which are $30 \mathrm{~cm}$ away from the emitter of the low frequency magnetic field. Typically, emitters represent the portable computers like a laptop or tablet. Furthermore, proposed measurement geometry has a universal approach. Hence, it can be used also for magnetic field emitters such as AC adapters. Unfortunately, the body position of the portable computer user may be closer than proposed measurement positions. Hence, investigation of an extremely low frequency magnetic field value at a smaller distance or in its center is mandatory from the users' point of view. The measuring positions proposed by the TCO standard for the objects emitting the magnetic field are given in Fig.7.

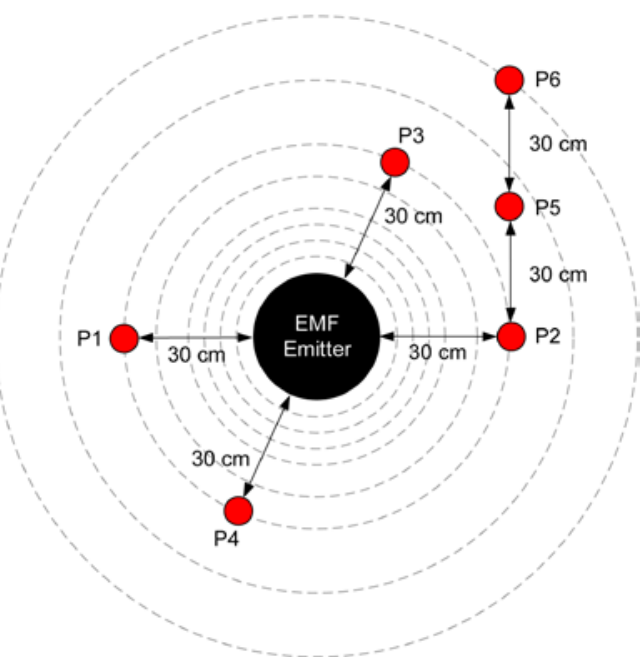

Fig.7. Measuring positions proposed by TCO standard for the objects that emit a magnetic field (P1, ..., P6).

\section{B. Tested AC adapter characteristics}

Table 5. shows the characteristics of the tested AC adapters, which include output voltage, output current and nominal power consumption.

Table 5. AC adapter characteristics.

\begin{tabular}{|c|c|c|c|}
\hline Laptop & Voltage [V] & Current [A] & Power [W] \\
\hline L1 & 19 & 6.32 & 125 \\
\hline L2 & 19 & 4.74 & 90 \\
\hline L3 & 19 & 3.95 & 75 \\
\hline L4 & 19 & 3.42 & 65 \\
\hline L5 & 19.5 & 4.62 & 90 \\
\hline L6 & 19 & 2.1 & 40 \\
\hline L7 & 19.5 & 4.62 & 90 \\
\hline L8 & 19.5 & 4.62 & 90 \\
\hline L9 & 19 & 2.37 & 45 \\
\hline L10 & 19 & 3.95 & 75 \\
\hline L11 & 19.5 & 4.62 & 90 \\
\hline L12 & 19 & 4.74 & 90 \\
\hline L13 & 19 & 4.74 & 90 \\
\hline L14 & 19 & 4.22 & 80 \\
\hline L15 & 15 & 5 & 75 \\
\hline L16 & 16.5 & 3.65 & 60 \\
\hline L17 & 19 & 2.37 & 45 \\
\hline
\end{tabular}




\section{ACKNOWLEDGMENT}

This work was partially supported by the Grant of the Ministry of Education, Science and Technological Development of the Republic Serbia [TR33037]. Authors are fully grateful to Ms. Janet Newell, native professor of English language, for her precious editing support.

\section{REFERENCES}

[1] IARC. (2002). IARC Monographs on the Evaluation of Carcinogenic Risks to Humans. Non-Ionizing Radiation, Part 1: Static and Extremely LowFrequency (ELF) Electric and Magnetic Fields. IARC Press, Volume 80.

[2] Advisory Group on Non-Ionising Radiation. (2001). ELF electromagnetic fields and the risk of cancer. Documents of the NRPB, 12 (1).

[3] Fernie, K.J., Reynolds, S.J. (2005). The effects of electromagnetic fields from power lines on avian reproductive biology and physiology: A review. Journal of Toxicology and Environmental Health, Part B: Critical Reviews, 8 (2), 127-140.

[4] World Health Organization (WHO). (2007). Extremely Low Frequency Fields: Environmental Health Criteria 238. WHO, ISBN 978-92-4-157238-5.

[5] Bastuji-Garin, S.R., Zittoun, R. (1990). Acute leukemia in workers exposed to electromagnetic fields. European Journal of Cancer, 26, 1119-1120.

[6] Harrington, J.M., McBride, D.I., Sorahan, T., Paddle, G.M., Van Tongeren, M. (1997). Occupational exposure to magnetic fields in relation to mortality from brain cancer among electricity generation and transmission workers. Occupational and Environmental Medicine, 54, 7-13.

[7] Johansen, C., Olsen, J. (1998). Mortality from amyotrophic lateral sclerosis, other chronic disorders and electric shocks among utility workers. American Journal of Epidemiology, 148, 362-368.

[8] Sobel, E., Dunn, M., Davanipour, Z., Qian, Z., Chui, H.C. (1996). Elevated risk of Alzheimer's disease among workers with likely electromagnetic field exposure. Neurology, 47, 1477-1481.

[9] Zoppetti, N., Andreuccetti, D., Bellieni, C., Bogi, A., Pinto, I. (2011). Evaluation and characterization of fetal exposures to low frequency magnetic fields generated by laptop computers. Progress in Biophysics and Molecular Biology, 107 (3), 456-463.

[10] Bellieni, C.V., Pinto, I., Bogi, A., Zoppetti, N., Andreuccetti, D., Buonocore, G. (2012). Exposure to electromagnetic fields from laptop use of "laptop" computers. Archives of the Environmental and Occupational Health, 67 (1), 31-36.

[11] TCO Development AB. (2012). TCO Certified Notebooks 4.0. http://tcodevelopment.com/files/2013/ 04/TCO-Certified-Notebooks-4.0.pdf.
[12] ICNIRP. (2010). Guidelines for limiting exposure to time-varying electric and magnetic fields $(1 \mathrm{~Hz}-100$ kHz). Health Physics, 99 (6), 818-836.

[13] Calvente, I., Fernandez, M.F., Villalba, J., Ilea, N. (2010). Exposure to electromagnetic fields and its relationship with childhood leukemia: A systematic review. Science of Total Environment, 408 (16), 3062-3069.

[14] Ott, H.W. (2009). Electromagnetic Compatibility Engineering. John Wiley \& Sons.

[15] Koppel, T., Ahonen, M. (2013). Radiofrequency electromagnetic fields from mobile devices used for learning and working. Elektronika ir Elektrotechnika, 19 (6), 65-70.

[16] Lutron Electronic Ent. Co., Ltd. Lutron EMF-828 specification. Www.sunwe.com.tw/lutron/EMF828eop.pdf.

[17] Aaronia AG. Aaronia Spectran NF-5035. http://www.aaronia.com/Datasheets/Spectrum_Analyz er/Gauss_Meter_Spectran_NF-5000.pdf.

[18] Futuremark Corporation. 3DMark Vantage. http://www.futuremark.com/benchmarks/3dmarkvantage.

[19] Brodić, D. (2015). Measurement of the extremely low frequency magnetic field in the laptop neighborhood. Revista Facultad de Ingeniería, 76, 39-45.

[20] Bradley, P.S., Mangasarian, O.L., Street, W.N. (1997). Clustering via concave minimization. In Advances in Neural Information Processing Systems 9 (NIPS 1996). NIPS Proceedings, Denver, CO, USA, 368-374.

[21] Mathews, J., Nair, M.S., Jo, L. (2012). Improved BTC algorithm for gray scale images using k-means quad clustering. In Neural Information Processing: 19th Int. Conference ICONIP 2012. Doha, Qatar, 9-17.

[22] Prasad, S., Kumar, P., Sinha, K.P. (2015). Grayscale to color map transformation for efficient image analysis on low processing devices. Advances in Intelligent Systems and Computing, 320, 9-18.

[23] Wang, H., Song, M. (2011). Ckmeans.1d.dp: Optimal $\mathrm{k}$-means clustering in one dimension by dynamic programming. The R Journal, 3 (2), 29-33.

[24] Brodic, D., Amelio, A. (2015). Classification of the extremely low frequency magnetic field radiation measurement from the laptop computers. Measurement Science Review, 15 (4), 202-209.

[25] Brodic, D. (2016). Analysis of the extremely low frequency magnetic field from laptop computers. Metrology and Measurement Systems, 23 (1), 143-154.

[26] Brodić, D., Amelio, A. (2016). Detecting of the extremely low frequency magnetic field ranges for laptop in normal operating condition or under stress. Measurement, 91, 318-341.

Received October 23, 2016. Accepted January 30, 2017. 